\title{
Correction: Kim, Y. K. et al. Tussilagone Inhibits the Inflammatory Response and Improves Survival in CLP-Induced Septic Mice. Int. J. Mol. Sci. 2017,
} 18,2744

\author{
Yun Kyu Kim ${ }^{1,3,+}$, Myeong Gu Yeo ${ }^{2,+}{ }^{+}$, Bo Kang $\mathrm{Oh}^{3}$, Ha Yeong Kim ${ }^{3}$, Hun Ji Yang ${ }^{3}$, \\ Seung-Sik Cho ${ }^{4}{ }^{(\mathbb{0}}$, Minchan Gil ${ }^{1, *}$ and Kyung Jin Lee ${ }^{3, *}$ \\ 1 Nano-Bio Resources Center, Department of Cosmetic Sciences, Sookmyung Women's University, \\ Seoul 04310, Korea; kingsagayo@gmail.com \\ 2 Department of Integrative Medical Sciences, Nambu University, Gwangju 506-706, Korea; \\ mgy11@nambu.ac.kr \\ 3 Department of Convergence Medicine, Asan Institute for Life Sciences, University of Ulsan College of \\ Medicine, Asan Medical Center, 88 Olympic-ro 43-gil, Songpa-gu, Seoul 05505, Korea; \\ bokang7804@gmail.com (B.K.O.); kimhayeong0516@gmail.com (H.Y.K.); didgnsw195@gmail.com (H.J.Y.) \\ 4 College of Pharmacy and Natural Medicine Research Institute, Mokpo National University, Muan, \\ Jeonnam 58554, Korea; sscho@mokpo.ac.kr \\ * Correspondence: minchangil@sookmyung.ac.kr (M.G.); kjlee@amc.seoul.kr (K.J.L.); \\ Tel.: +82-2-2077-7717 (M.G.); +82-2-3010-4030 (K.J.L.); Fax: +82-2-6359-7006 (M.G.); +82-3010-4147 (K.J.L.) \\ + These authors contributed equally to this work.
}

Received: 17 December 2019; Accepted: 23 December 2019; Published: 25 December 2019 updates

We wish to make the following corrections to this paper [1]:

We found that Figure 7A,C data were unintentionally reused from the previously published data [2]. The mistake happened during the preparation of data figures for the revision in the peer-review process. All authors regret that error.

Due to the incorrect figure in original Figure 7A,C, replace the following

A
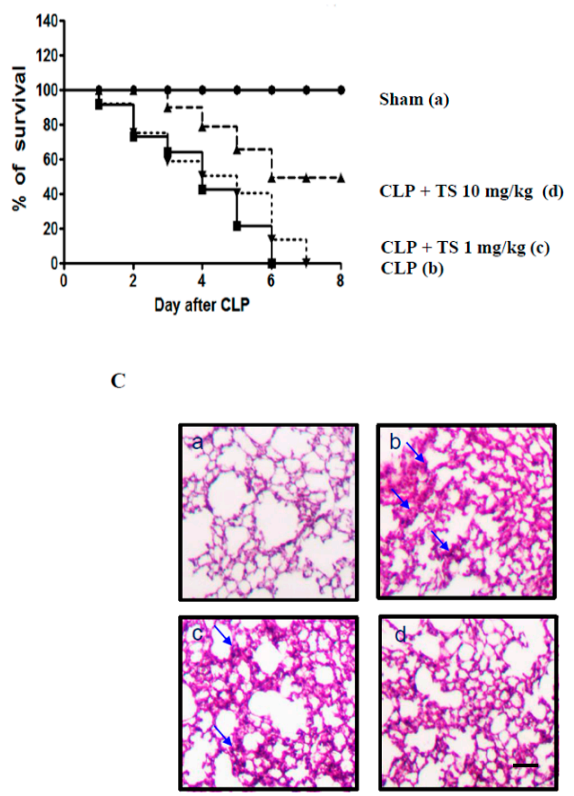

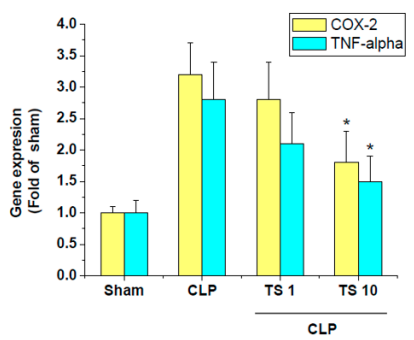

D

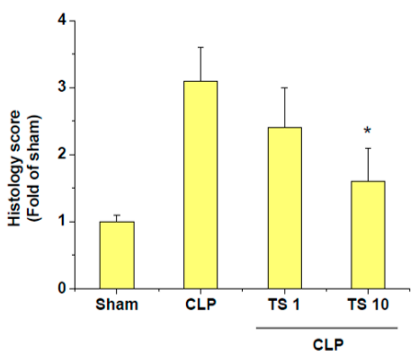


with the corrected Figure 7 (Figure 1)

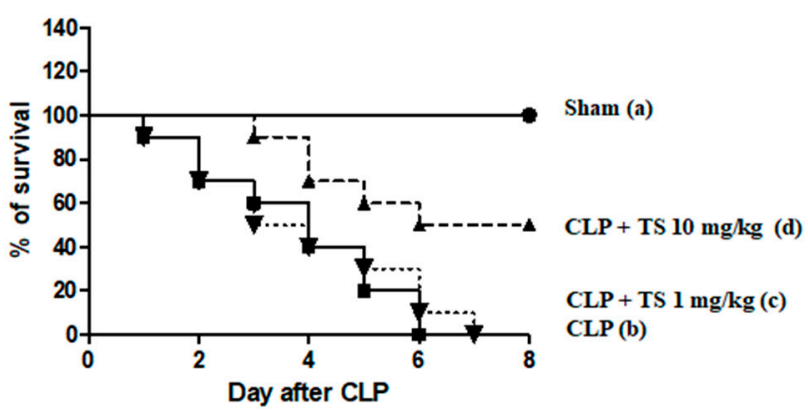

C

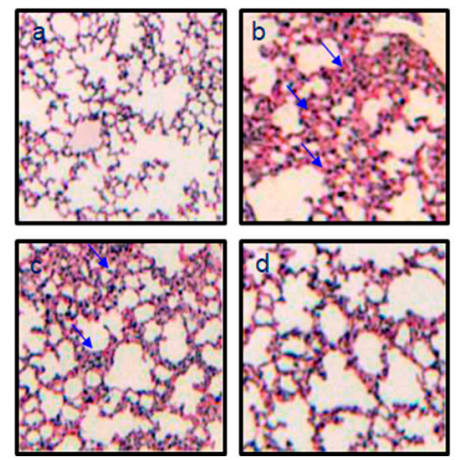

B

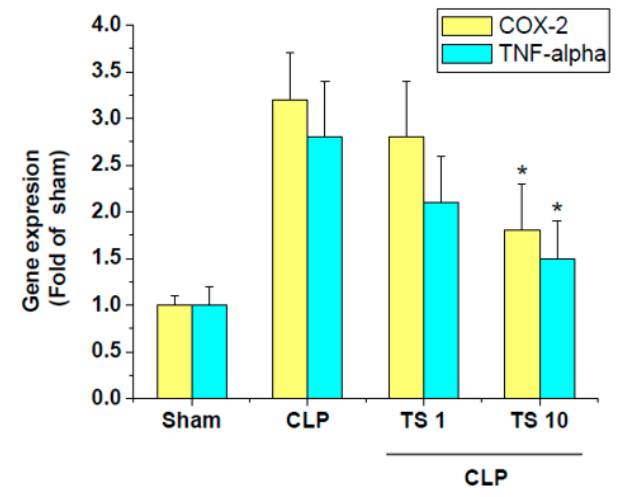

D

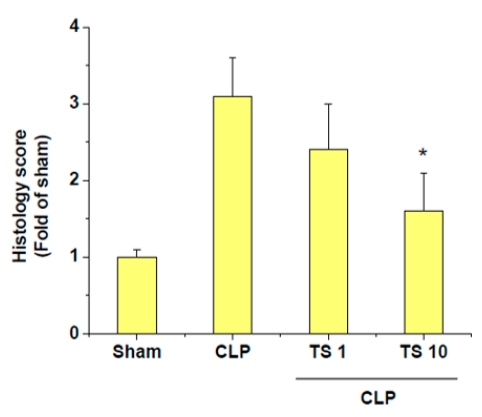

Figure 1. Effect of TS on survival and lung injury in cecal ligation and puncture (CLP)-induced septic mice. (A) To examine the effect of TS on the survival of CLP-induced septic mice, survival of mice was then monitored every $24 \mathrm{~h}$ for up to 8 days for the following experimental groups (a) sham control; mice were orally administered with either (b) vehicle (corn oil, $0.1 \mathrm{~mL}$ per mouse, $n=5$ ), (c) $1 \mathrm{mg} / \mathrm{kg}$ TS $(n=5)$, or $(\mathrm{d}) 10 \mathrm{mg} / \mathrm{kg}$ TS $(n=5), 2 \mathrm{~h}$ prior to the operation. Significantly different from CLP-induced septic group (B) Expression of COX-2 and TNF- $\alpha$ transcripts in the isolated PAM were determined by real-time PCR; ${ }^{*} p<0.05$ vs. CLP-induced septic group ( $n=3$ in each group) (C) The lungs from each experimental group were processed for histologic evaluation 1 day after CLP. Representative histologic changes in lung tissue obtained from mice belonging to each group are displayed and the arrows indicate the damaged area (hematoxylin and eosin staining; magnification $400 \times$ ). Scale bar represents $200 \mathrm{um}$. (D) The extent of lung injury was estimated using scores in different sections for neutrophil infiltration, hemorrhage, necrosis, congestion, and edema. ${ }^{*} p<0.05$ vs. CLP-induced septic group ( $n=3$ in each group).

We would like to apologize for any inconvenience caused to the readers by these changes.

Conflicts of Interest: The authors declare no conflict of interest. 


\section{References}

1. Kim, Y.K.; Yeo, M.G.; Oh, B.K.; Kim, H.Y.; Yang, H.J.; Cho, S.S.; Gil, M.; Lee, K.J. Tussilagone Inhibits the Inflammatory Response and Improves Survival in CLP-Induced Septic Mice. Int. J. Mol. Sci. 2017, 18, 2744. [CrossRef] [PubMed]

2. Gil, M.; Kim, Y.K.; Hong, S.B.; Lee, K.J. Naringin Decreases TNF- $\alpha$ and HMGB1 Release from LPS-Stimulated Macrophages and Improves Survival in a CLP-Induced Sepsis Mice. PLoS ONE 2016, 11, e0164186. [CrossRef] [PubMed] 